\title{
FLUCTUATIONS IN THE MICROWAVE SKY
}

\author{
R. B. PARTRIDGE
}

Haverford College

\begin{abstract}
This paper reviews the great progress recently made in searches for and the characterization of anisotropies in the cosmic microwave background. We now have secure detections on some angular scales and improved upper limits on others. As the sensitivity of such searches increases, understanding sources of foreground noise (e.g., Galactic and extragalactic radio emission) becomes more important. Also reviewed are the contributions aperture synthesis (interferometric) observations can make in characterizing cosmic background fluctuations and foreground sources of noise. Some recent results from the Very Large Array at $\lambda=3.6 \mathrm{~cm}$ are given; these set a limit $\Delta \mathrm{T} / \mathrm{T} \lesssim 1.4 \times 10^{-5}$ on fluctuations at $\theta \sim 80^{\prime \prime}$. Recent work on the Sunyaev-Zel'dovich effect is summarized.
\end{abstract}

The cosmic microwave background radiation (CBR), the remnant of the hot Big Bang phase of the Universe, is the best studied of all cosmic backgrounds. In the 5 years since the last IAU symposium dealing with background radiation fields (IAU Symposium 139 , held in Heidelberg), there has been startling progress in studies of the CBR. Other papers in this volume will present the results of much of this recent work. In my contribution, I wish to reflect a bit on the change of mood in the field of CBR studies produced by these recent observational successes, then reemphasize the importance of a clear understanding of foreground sources of noise, and finally to highlight the importance of interferometry as a technique in the study of the angular distribution of the CBR. In the course of the last of these three, I will present some new observational work in which I was involved.

When I reviewed the CBR at Symposium 139, I stressed just how featureless that background is. The spectrum is thermal and, in 1989, no anisotropy had been reliably detected with the exception of the dipole moment due to the motion of the earth. The first of these statements is still true; there is no evidence for spectral distortions of any sort in the spectrum of the CBR, and upper limits on various classes of spectral perturbations have been sharply improved, as summarized by John Mather here. The second situation has changed radically. Anisotropy on a range of angular scales has now been detected or reported. The first such report was by the COBE group (Smoot et al., 1992), but other papers have followed quickly on the heels of that breakthrough letter. The fact that we do now have detections rather than increasingly stringent upper limits has produced a mental phase change in the field. Dare I refer to the work of Thomas Kuhn and call it a "paradigm shift"? Formerly, those of us who spent decades working on the CBR aimed to set tighter and tighter upper limits on the amplitude $\Delta \mathrm{T} / \mathrm{T}$ of fluctuations in the CBR on various angular scales. The aim of reducing limits on $\Delta \mathrm{T} / \mathrm{T}$ may have led some of us on occasion to discard or downplay some real signals. The situation is different now-we are now trying to measure an effect known

59

M. Kafatos and Y. Kondo (eds.), Examining the Big Bang and Diffuse Background Radiations, 59-70. (c) 1996 IAU. Printed in the Netherlands. 
to exist. I would suggest that some of the confusion in the field, particularly the apparently discordant results of those groups working on scales $\theta \sim 1^{\circ}$, may be due to the understandable turmoil induced by this paradigm shift.

There is another consequence of this change in mood. Formerly, if we encountered a foreground source of noise we did not fully understand, we were safe in lumping it in with the signal when deriving upper limits on $\Delta T / T$. Now that we have detections, we may no longer do so. We must understand the backgrounds in order to correct actual measurements. For just that reason, I will discuss here foreground emission, both Galactic and extragalactic.

\section{1.) Summary of Recent Results}

Let me begin with a brief survey of the observational status of searches for anisotropies in the CBR. On scales of $\sim 10^{\circ}$ and above, we have an excellent all-sky map provided by the DMR instruments aboard COBE (see Smoot's paper in this volume). Those important satellite results have been confirmed by a balloon-borne experiment (Ganga et al., 1993) and more recently by a ground-based experiment at comparable angular scales but lower frequencies (Hancock et al., 1994; Davies et al. in this volume). The cross-correlation of the results of Hancock et al. with the COBE map allows us to identify regions of real high and low temperature, as well as to calculate rms values of $\Delta \mathrm{T} / \mathrm{T}$. We thus do now have real "pictures" of the microwave sky on angular scales of $\sim 10^{\circ}$.

The single most active area in observational CBR studies is the search for anisotropies on scales of approximately $1^{\circ}$. Several groups are involved in observations from the ground, generally at the South Pole, or from balloon-borne instruments. The work of some of those groups, at least, will be reviewed by Lubin in this volume. While I believe it is safe to say that fluctuations on degree scales have indeed been detected, the experimental situation is not completely clear, and some of the results appear to be inconsistent as of the summer of 1994. Some groups report only upper limits (e.g. the work of Tucker et al., 1993, on a scale of $0.15^{\circ}$, and the work of Schuster et al., 1993, on a scale approximately ten times larger); other groups report clear detections (e.g. Wollack et al., 1993; Piccirillo and Callisse, 1993; Cheng et al., 1994; deBernardis et al., 1994; Dragovan et al., 1994, all on intermediate scales). As a rough rule of thumb, however, I believe it is fair to claim that $\Delta \mathrm{T} / \mathrm{T}$ is likely to fall in the range $1.5-5 \times 10^{-5}$.

On scales $\sim 0.1^{\circ}$, that is a few arcminutes, the best results were from the Owens Valley Radio Observatory (OVRO) until very recently. This group performed two different experiments at a wavelength of $1.5 \mathrm{~cm}$, with a beam size of $1.5^{\prime}$. Both sets of observations were made near the north celestial pole. The first (Readhead et al., 1989) traced out a small circumpolar arc with seven independent samples. The beam switch angle employed was $7.15^{\prime}$, and the resulting upper limit on $\Delta \mathrm{T} / \mathrm{T}$ was $1.7 \times 10^{-5}$ (see Fig. 1). The second program (Myers et al., 1993) fully sampled a larger circumpolar ring, with each point receiving less integrating time. More points were sampled, but the limit on $\Delta \mathrm{T} / \mathrm{T}$ is lower: $\leq 10^{-4}$.

Very recently, results from the Ryle telescope at Cambridge have begun to come in (Jones et al., 1993, 1994; Saunders, 1994). Using interferometric techniques, they have mapped the Sunyaev-Zel'dovich signal in several clusters of galaxies with $100 \mu \mathrm{K}$ 
accuracy. The resolution used for these studies is $\sim 80^{\prime \prime}$, and the instrument has the potential to work at both higher resolution and somewhat higher sensitivity, i.e. to be able to detect $\Delta \mathrm{T} / \mathrm{T} \sim 1-3 \times 10^{-5}$ fluctuations. On scales below a few arcminutes, as we shall see, the technique of choice is also interferometry, and I will report on that more fully in $\$ 3$ below.

Before proceeding, I want to draw a few conclusions from the work I've summarized above. The first is that COBE and some of the other experiments have detected fluctuations, and the amplitudes of these fluctuations are, to within an all important factor of 2 or so, consistent with CDM models for structure formation and with a spectral index of the initial density perturbations $n=1$. The Tenerife results do hint at a value for this index $n$ slightly greater than unity. There is no evidence (yet) for secondary ionization which would damp the fluctuations at $0.1^{\circ}-1^{\circ}$ (Ostriker and Vishniac, 1986; Bond et al., 1991; but see also Tegmark, Silk and Blanchard, 1994) or for cosmic strings (Bouchet et al., 1988). The Sunyaev-Zel'dovich effect in nearby clusters has been detected (e.g. Birkinshaw et al., 1994; and Jones, 1994), and in some cases we are able to calculate a value for Hubble's constant using the technique first noted by Gunn (1978): $\mathrm{H}_{0} \sim 40 \mathrm{~km} / \mathrm{sec}$ per Mpc. It is worth noting that in order to obtain a larger value for $\mathrm{H}_{0}$, one would need to show that values of $\Delta T / T$ are substantially smaller than currently reported.

\section{2.) Foreground Sources of Noise}

As I have noted, now that we have actual detections we need to understand and correct for foreground sources of noise that can mimic or mask $\Delta T / T$ fluctuations in the CBR. There are two kinds of error, statistical (errors that increase the size of the individual error bars in a plot like Fig. 1) and systematic (those that increase the scatter of the points in a diagram like Fig. 1). Needless to say, it is systematic errors that cause the most concern.

Now let me list briefly sources of foreground error. First, there is pickup of emission from the ground or from other nearby "room temperature" sources. This can create both statistical and systematic error; systematic if the amplitude of ground pickup depends on the position of the telescope (see for instance Perrenod and Lada, 1979, or Lake and Partridge, 1980). A second source, primarily of statistical noise, is emission from the earth's atmosphere. To reduce atmospheric noise, many groups now work at the South Pole, at an effective altitude of $\sim 3000$ meters, or use balloons to carry their equipment above most of the atmosphere. I shall focus in this paper on patchy emission from our Galaxy, which has the potential for creating substantial systematic errors, and on emission from extragalactic radio sources which of course contributes to the variance of the microwave sky, and hence to systematic errors as defined above.

\section{Galactic Emission}

Microwave and millimeter wave emission from our Galaxy is now seen as the most troublesome source of foreground noise and the primary limit to improved measurements of CBR fluctuations. At wavelengths $\gtrsim 3-5 \mathrm{~mm}$, bremsstrahlung and 


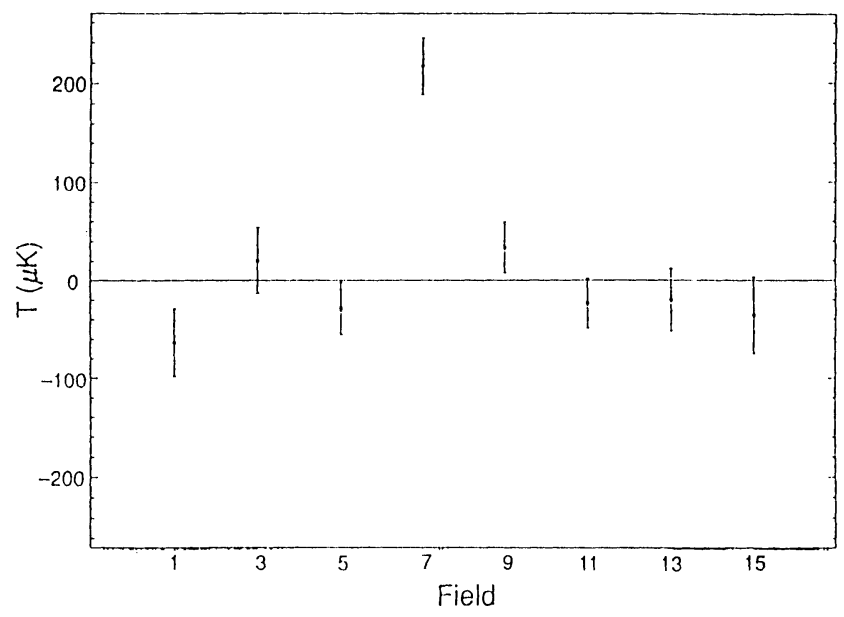

Fig. 1. Results of a typical search for fluctuations in the CBR (in this case, the work of Readhead et al., 1989, with permission). Statistical errors, as defined here, increase the size of the error bars (and may also, therefore, increase the point-to-point scatter). Systematic errors instead increase the point-to-point scatter only.

synchrotron radiation dominate, with spectra $T(v) \propto v^{-2.1}$ and $v^{-2.8}$, respectively. At wavelengths $\lesssim 3-5 \mathrm{~mm}$, re-emission from Galactic dust with $\mathrm{T} \sim 24 \mathrm{~K}$ dominates; here $T(v) \propto v^{1.6}$ in the Rayleigh Jeans region (Bennett, et al., 1992; Toffolatti, et al., 1994).

Our interest is in the spatial fluctuations in foreground Galactic noise as a function of frequency and angular scale. Such calculations are done either for specific regions of the sky (e.g. Banday and Wolfendale, 1991a, b) using other astronomical data, such as low frequency radio maps (Haslam et al., 1982; Lawson, et al., 1987), or "generically" for typical regions of the sky (see, e.g. Banday and Wolfendale, 1990; Brandt et al., 1994; Partridge, 1994). Most of the work has concentrated on degree scales. The VLA observations to be described below permit us to derive "generic" constraints on $\theta \sim 0.01^{\circ}$ scales as well. Typical "generic" limits for $\theta \sim 1^{\circ}$ are shown in Fig. 2 below, adopted from the useful recent review by Danese et al. (1994) and from my 1994 book. 


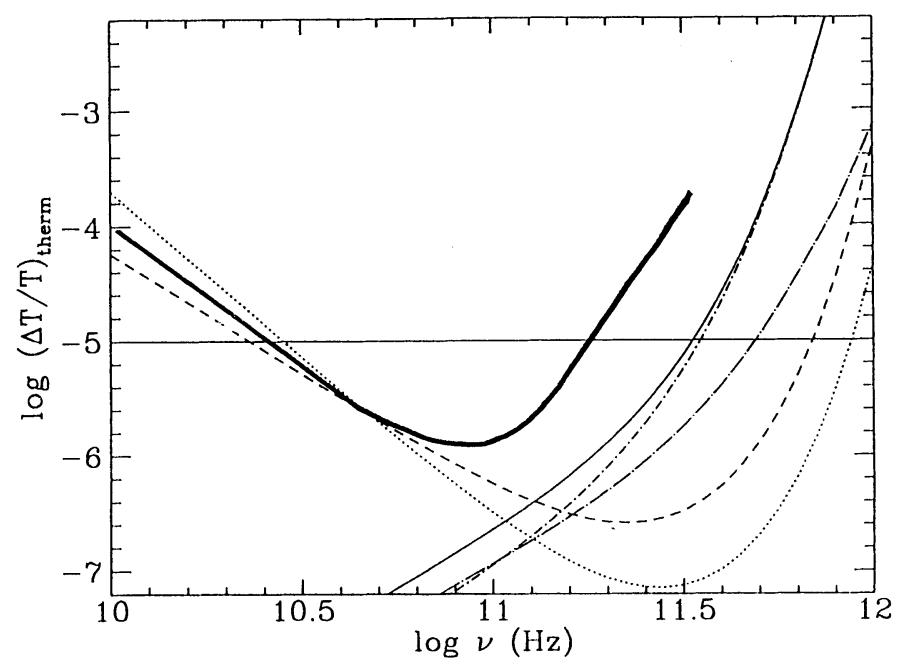

Fig. 2. Models for Galactic foreground noise on $\sim 1^{\circ}$ scales. The thin lines are models of Danese et al. (1994), and represent synchrotron fluctuations (dotted) and free-free emission (dashed) at low $V$, and dust re-emission at high $v$ (solid). The two dot-dash lines show the contributions from hot and cool dust. The heavy lines are comparable models from my 1994 book. My (very conservative) model for dust emission assumes up to $100 \%$ amplitude fluctuations in the dust emission.

\section{Extragalactic Foregrounds}

This problem has been extensively investigated by the Padua group; see, for instance, Franceschini et al. (1989) and Danese et al. (1994). At wavelengths greater than a centimeter or so, substantial point-to-point fluctuations are introduced by radio galaxies and QSO's. At shorter wavelengths, typically a few millimeters or below, it is the emission from dusty galaxies (e.g. IRAS galaxies) that will dominate. As the work of Franceschini et al. shows, that leaves a nice wavelength "window" for such observations: see Fig. 3, adopted from their work.

In order to estimate the fluctuation level at various angular scales and various frequencies, both the counts (number per square degree) and the spectra of different classes of radio sources are needed. At wavelengths greater than, say, $3 \mathrm{~cm}$, both are well known (see Wall here; Gregory and Condon, 1991; and Windhorst et al., 1993). Thus the left half of Fig. 3 is fairly well understood. The right half of the diagram is considerably less certain. Source counts are more difficult at higher frequencies; spectra are less well known, and large k-corrections are needed for sources at substantial redshifts because of the positive spectral index of dust emission. 


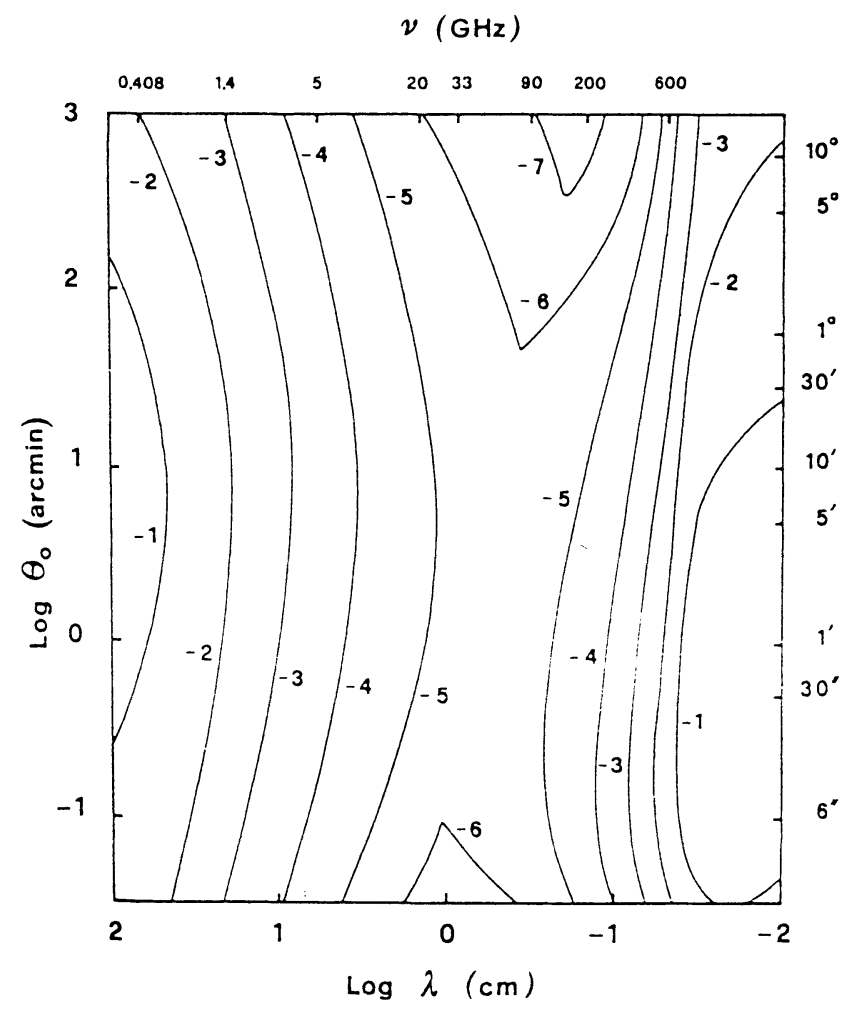

Fig. 3. Estimates of the rms fluctuation level introduced by foreground extragalactic radio sources (from Franceschini et al., 1989). In this generic model, contours of $\Delta \mathrm{T} / \mathrm{T}$ are shown as a function of observing scale and wavelength.

For measurements on degree scales and above, I would claim, our knowledge of the distribution and spectra of radio sources is probably good enough to allow us to eliminate foreground noise due to radio galaxies and QSO's; for instance, use of the formula $S=2 \mathrm{kT} \Omega / \lambda^{2}$, with $\Omega=1.13 \theta_{1 / 2}^{2}$ for a gaussian beam, allows us to derive the following limit on the flux density of a source which will produce a $\leq 10 \mu \mathrm{K}$ signal in observations made with a beam of $\theta$ degrees full width at half-maximum:

$$
S_{c}=0.1\left(\frac{\lambda}{1 \mathrm{~cm}}\right)^{-2}\left(\frac{\theta}{1^{\circ}}\right)^{2} .
$$


Note that the limiting value of $S$ is proportional to the square of the beam half-power width. Sources with flux density above $0.1 \mathrm{Jy}$ can easily be detected by survey instruments so that their contributions can be removed.

The spectrum of most ordinary synchrotron radio sources decreases rapidly with frequency; that is the reason for the sharp falloff in frequency in the left half of Fig. 3. There are, however, occasional sources with inverted spectra; that is, sources whose flux density is $\alpha v^{\alpha}$ with $\alpha>0$. Among these is a class of sources called the gigahertz peaked spectrum sources (see O'Dea, Baum and Stanghellini, 1991). They are, fortunately, rare; and as radio surveys have been pushed to higher frequency, no new population of sources with strongly inverted spectra has so far turned up (see recent surveys such as Aizu et al., 1987; Donnelly et al., 1987; Gregory and Condon ,1991; and Windhorst et al., 1993).

Now for a nightmare scenario, suppose one of these gigahertz peaked spectrum sources does lie in an area studied for CBR fluctuations. Furthermore, suppose it is variable in time so that follow-up observations made elsewhere can not necessarily reveal its flux at the time the CBR measurements were made. That would be truly unpleasant! A year or so ago, purely serendipitously, my colleagues and I discovered a gigahertz peaked spectrum source whose low frequency spectrum was indistinguishable from that of a blackbody. At 3.6 and $6 \mathrm{~cm}$, its flux density was of order $0.2 \mathrm{Jy}$, i.e. bright enough to cause problems in degree scale experiments, if those experiments had been made at centimeter wavelengths. Fortunately, the spectrum was later confirmed to fall off rapidly at higher frequencies. Thanks to observations kindly made at the Ryle Telescope by Mike Jones and his colleagues, we know that the $2 \mathrm{~cm}$ flux is only $0.07 \mathrm{Jy}$, and the Cambridge observations also suggest that the source has not been substantially variable in the past year. It nevertheless provides a warning. Radio surveys are and have been carried out at wavelengths an order of magnitude or more larger than most searches for CBR fluctuations. The properties of extragalactic radio sources-counts, spectra and variability-at mm wavelengths are less well known than we would like. Nor will better data be easy to obtain. Fortunately, since $\Delta \mathrm{T} \propto \lambda^{2}$ for a fixed value of $S$, we need be concerned only with quite bright $\mathrm{mm}$ wave sources, which can be detected and monitored with available radio telescopes.

\section{3.) Interferometric Observations}

It has been recognized for some time that interferometric or aperture synthesis observations of the CBR offer a number of advantages in addition to high angular resolution (see Thompson et al., 1986; or for specific reference to the CBR, Partridge, 1994 , and Saunders, 1994). Systematic errors contributed by emission from the ground and from the atmosphere are largely canceled out in interferometric observations, for instance (see Knoke et al., 1984; Timbie and Wilkinson, 1990).

Until fairly recently, interferometric observations of the CBR were made primarily on sub-arcminute scales (Fomalont et al., 1988; Martin and Partridge, 1988; Hogan and Partridge, 1989; Fomalont et al., 1993) to probe the CBR angular spectrum on scales likely to reveal the effects of reionization (Vishniac, 1987), explosive galaxy formation (Ostriker and Cowie, 1981), or cosmic strings (Bouchet et al., 1988). The advantages of such observations are now being realized in larger-scale observational programs as 
well. The pioneering effort was by Timbie and Wilkinson (1990) using a two-element array, with a very short spacing that provided angular resolution of $\sim 1^{\circ}$. More sophisticated, multi-element arrays have been constructed by the Cambridge group, and I will report some of their results below. Let me first summarize our own work at $10^{\prime \prime}-80^{\prime \prime}$ scales.

\section{3a.) Observations at the Very Large Array (VLA)}

The as-yet unpublished observational results reported in this section were obtained in collaboration with Ed Fomalont and Ken Kellermann of NRAO, Eric Richards of Haverford College and Rogier Windhorst of Arizona State University.

We employed the VLA (Very Large Array) at $3.6 \mathrm{~cm}$ wavelength to make a series of deep maps of the microwave sky, each spanning an area of $\sim 5^{\prime}$ in radius. In the first round of observations (Fomalont et al., 1993; Windhorst et al., 1993), we amassed $\sim 80$ hours of observations on two fields with the VLA in its D configuration, for which the resolution was 10". These results are presented in the papers listed above.

The second round of observations was a further 100 hours of VLA time in the $\mathrm{D}$ configuration on a single field included in the Hubble Space Telescope Medium Deep Survey (Griffiths et al., 1994). The observed rms noise of our final map was $1.97 \mu \mathrm{Jy}$, making it the most sensitive radio image ever made. We identified 28 sources exceeding $9 \mu \mathrm{Jy}$ or $4.5 \sigma$. The brightest has $\mathrm{S}=278 \mu \mathrm{Jy}$. Many are now optically identified; see below.

In the months following IAU Symposium 168, my colleagues and I have made additional observations of the same region using the VLA in the C configuration, with $\sim 3$ " resolution. The higher resolution will improve our sensitivity for the detection of discrete sources and will substantially improve the accuracy of the radio source positions; the latter in turn will make our optical identifications more certain. Richards, Spillar and I are also making follow-up $2 \mu$ observations of some of these optical sources at the Wyoming Infrared Observatory.

We have not yet analyzed our most recent data. Preliminary results from the first $3.6 \mathrm{~cm}$ runs reveal no evidence for CBR fluctuations, with a $95 \%$ confidence level upper limit of $\sim 1.4 \times 10^{-5}$ on $\Delta \mathrm{T} / \mathrm{T}$ at $1^{\prime}$ scale, i.e. $~ 30 \%$ lower than our published upper limit (Fomalont et al., 1993). It is our hope that additional observations now underway will allow us to lower this preliminary upper limit. The absence of detectable CBR fluctuations can set interesting constraints on the evolution of clusters of galaxies (e.g., Partridge, 1995a) and on cosmic strings. Our upper limits correspond to a limit $\mu \lesssim$ few $\times 10^{22} \mathrm{gm} / \mathrm{cm}$ on strings; that value in turn sets an approximate limit of $\sim 10^{16} \mathrm{GeV}$ on the energy scale of spontaneous symmetry breaking (Partridge, 1995b). The absence of detectable small-scale fluctuations is also consistent with the absence of re-ionization.

Our most recent $3.6 \mathrm{~cm}$ map is of a region at $\alpha=13^{\mathrm{h}} 12^{\mathrm{m}}, \delta=+42^{\circ} 38^{\prime}$, lying within the area of the WFPC Medium Deep Survey (Griffiths, et al., 1994) made by the Hubble Space Telescope. We thus have high resolution optical images for many of the 28 radio sources identified in the map. (The radio detection threshold was set at $4.5 \sigma$ or $9 \mu \mathrm{Jy}$.) Of 16 radio sources lying within the WFPC frames, 14 are robustly identified optically. Two are quasars and the other 12 faint, often blue, galaxies, several of which are in 
small groups or show morphological evidence of mergers and other peculiarities. To optical limits of 23.2 in V and $21^{\mathrm{m}} \cdot 9$ in I, most of the optically identified galaxies are radio sources. The $C$ configuration observations now underway should allow us to improve our identification statistics as well as to explore the radio morphology of identified sources. All of these results will be discussed in more detail in a paper submitted to Nature (Windhorst et al., 1995), and in a future, longer paper.

\section{$3 b$ Recent work at the Ryle Telescope}

Mike Jones of the Mullard Radio Astronomy Observatory, Cambridge, kindly provided me with some results in advance of publication (see Jones et al., 1993; also Jones, 1994; Saunders, 1994). These were obtained with the Ryle Telescope, an interferometer consisting of eight elements operating at $\lambda=2 \mathrm{~cm}$. For these observations, the resolution was $80^{\prime \prime}$; scales from $\sim 2^{\prime \prime}$ to $\sim 2^{\prime}$ could be probed with reasonable sensitivity.

The instrument was used first to search for and characterize the Sunyaev-Zel'dovich (SZ) signal from several nearby clusters of galaxies. The Sunyaev-Zel'dovich (1972) effect arises from inverse Compton scattering of CBR photons from the electrons in the intergalactic plasma in clusters. In the Rayleigh-Jeans region of the CBR spectrum, the observed magnitude of the temperature decrement is proportional to $n_{e} T_{e} \ell$, where $n_{e}$ and $T_{e}$ are the electron density and temperature, and $\ell$ is the path length through the cluster.

Fig. 4 shows one of the Ryle results, for cluster Abell 2218. The detection of the SZ signal is robust, and the results agree with earlier work at "conventional" filled-aperture telescopes (see Birkinshaw, 1991, for a review).

As Gunn (1978) among others suggested, a measurement of the SZ effect combined with a measurement of the X-ray luminosity of the same cluster $\left(\propto \mathrm{n}_{\mathbf{e}}^{2} \mathrm{~T}{ }_{\mathbf{e}}^{1 / 2}\right)$ provides in principle an independent means of finding the distance to the cluster, and hence determining $\mathrm{H}_{0}$. It is interesting that Jones (1994), on the basis of the Ryle Telescope observations, finds a value of $\mathrm{H}_{0}$ in better agreement with $\mathrm{H}_{0} \sim 50 \mathrm{~km} / \mathrm{sec}$ per Mpc than with 100 in the same units. Similar, low, values of $\mathrm{H}_{0}$ have been found by others as well (e.g. Birkinshaw et al., 1991; Herbig et al., 1994) using this method.

I emphasize that these results are preliminary. The power of interferometric observations of the CBR has not yet been fully realized. By the time this volume appears, substantially better results may have been obtained at the Ryle Telescope and elsewhere. And an even more intriguing instrument, the Very Small Array, may finally be under construction. This instrument, long planned by the Cambridge group, is an array of horn antennas specifically designed to detect CBR fluctuations on $\sim 3^{\prime}-30^{\prime}$ scales, which encode most of the cosmologically interesting information in the angular spectrum.

\section{Acknowledgments}

My research has been supported in part by two grants from the National Science Foundation, AST 89-14988 and AST 93-20049. 


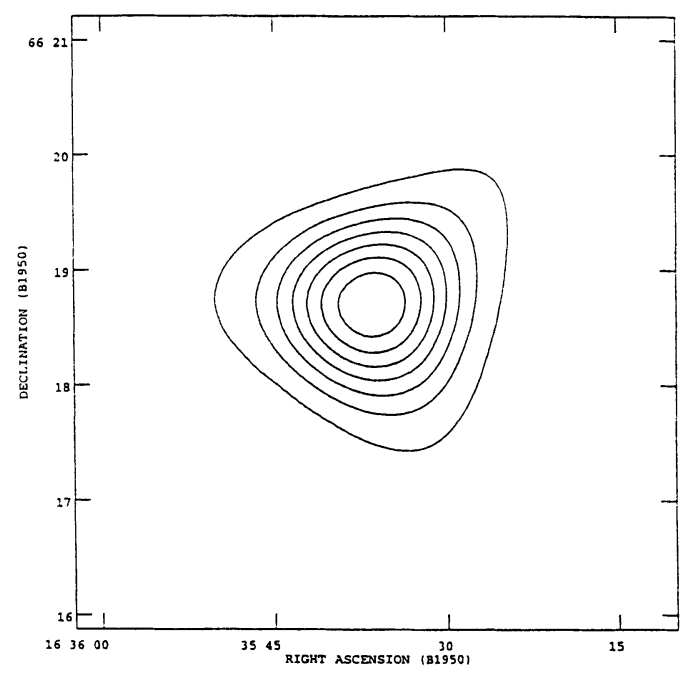

Fig. 4. Map of the SZ effect in Abell 2218, made by the Ryle Telescope (courtesy M. Jones). The contour levels range from $-2 \mu \mathrm{Jy}$ to $-26 \mu \mathrm{Jy}$. The "bulge" to the east is present in ROSAT X-ray maps as well.

\section{References}

Aizu, K., Inoue, M., Tabara, H., and Kato, T. 1987 in IAU Symposium 124, Observational Cosmology, ed. A. Hewitt, G. Burbidge, and L. Z. Fang, Reidel Publ. Co., Dordrecht, the Netherlands.

Banday, A. J., and Wolfendale, A. W. 1990, Monthly Not. Roy. Astr. Soc., 245, 182.

Banday, A. J., and Wolfendale, A. W. 1991a, Monthly Not. Roy. Astr. Soc., 252, 462.

Banday, A. J., Giler, M., Szabelska, B., Szabelski, J., and Wolfendale, A. W. 1991b, Ap. J., 375,432 , and references therein.

Bennett, C. L. et al. 1992, Ap. J. (Letters), 396, L7.

Birkinshaw, M. 1991, in Physical Cosmology, eds. A. Blanchard et al., Editions Frontiers, Gif-sur-Yvette, France.

Birkinshaw, M., Hughes, J. P., and Arnaud, K. A. 1991, Ap. J. 379, 466.

Birkinshaw, M., and Hughes, J. P. 1994, Ap. J. 420, 33.

Bond, J. R., Carr, B. J., and Hogan, C. J. 1991, Ap. J. 367, 420.

Bouchet, F. R., Bennett, D. P., and Stebbins, A. 1988, Nature, 335, 410.

Brandt, W. N., et al. 1994, Ap. J. 424, 1.

Cheng, E. S., et al. 1994, Ap. J. (Letters) 422, L37.

Danese, L. et al. 1994, Astrophys. Letters and Communications, in press.

de Bernardis, P., et al. 1994, Ap. J. (Letters) 422, L33.

Donnelly, R. H., Partridge, R. B., and Windhorst, R. A. 1987, Ap. J., 321, 94. 
Dragovan, M. et al. 1994, Ap. J. 427, L67.

Fomalont, E. B., Kellermann, K. I., Anderson, M. C., Weistrop, D., Wall, J. V., Windhorst, R. A., and Kristian, J. A. 1988, A. J., 96, 1187.

Fomalont, E. B., Partridge, R. B., Lowenthal, J. D., and Windhorst, R. A. 1993, Ap. J., 404, 8.

Franceschini, A., Toffolatti, L., Danese, L., and De Zotti, G. 1989, Ap. J., 344, 35.

Ganga, K., Cheng, E., Meyer, S., and Page, L. 1993, Ap. J. (Letters) 410, L57.

Gregory, P. C., and Condon, J. J. 1991, Ap. J. Suppl. 75, 1011.

Griffiths, R. E. et al. 1994, Ap. J. 437, in the press.

Gunn, J. E. 1978, in Observational Cosmology, ed. A. Maeder, L. Martinet, and G. Tammann (Geneva: Geneva Observatory), p. 3.

Hancock, S., Davies, R. D., Lasenby, A. N., Guttierez de la Cruz, C. M., Watson, R. A., Rebolo, R., and Beckman, J. E. 1994, Nature 367, 333.

Haslam, C. G. T., Salter, C. J., Stoffel, H., and Wilson, W. E. 1982, Astron. and Astrophys. Suppl., 47, 1.

Herbig, T., Lawrence, C. R., Readhead, A. C. S., and Gulkis, S. 1994, Ap. J. (Letters), submitted.

Hogan, C., and Partridge, R. B. 1989, Ap. J. (Letters), 341, L29.

Jones, M. 1994, Astrophys. Letters and Commun., in press.

Jones, M. et al. 1993, Nature 365, 320.

Knoke, J. E., Partridge, R. B., Ratner, M. I., and Shapiro, I. I. 1984, Ap. J., 284, 479.

Lake, G., and Partridge, R. B. 1980, Ap. J., 237, 378.

Lawson, K. D., Mayer, C. J., Osborne, J. L., and Parkinson, M. L. 1987, Monthly Not. Roy. Astr. Soc. 225, 307.

Martin, H. M. and Partridge, R. B. 1988, Ap. J., 324, 794.

Myers, S. T., Readhead, A. C. S., and Lawrence, C. R. 1993, Ap. J. 405, 8.

O'Dea, C. P., Baum, S. A., and Stanghellini, C. 1991, Ap. J. 380, 66.

Ostriker, J. P., and Cowie, L. L. 1981, Ap. J. (Letters), 243, L127.

Ostriker, J. P. and Vishniac, E. T. 1986, Ap. J. (Letters), 306, L51.

Partridge, R. B. 1994, 3K: The Cosmic Microwave Background Radiation, Cambridge Univ. Press, Cambridge.

Partridge, R. B. 1995a, in Extragalactic Background Radiation, ed. M. Livio et al.

Partridge, R. B. 1995b, in Non-Accelerator Particle Physics, eds. R. Cowsik and K. R. Sivaraman, World Scientific Publ. Co., Singapore.

Perrenod, S. C., and Lada, C. J. 1979, Ap. J. (Letters), 234, L173.

Piccirillo, L., and Calisse, P. 1993, Ap. J. 411, 529.

Readhead, A. C. S., Lawrence, C. R., Myers, S. T., Sargent, W. L. W., Hardebeck, H. E., and Moffet, A. T. 1989, Ap. J., 346, 566.

Saunders, R. 1994, Astrophys. Letters and Commun., in press.

Schuster, J. et al. 1993, Ap. J. (Letters) 412, L47.

Smoot, G. F., et al. 1992, Ap. J. (Letters), 396, L1.

Tegmark, M., Silk, J., and Blanchard, A. 1994, Ap. J. 420, 484, and erratum in Ap. J. 434, 395.

Thompson, A. R., Moran, J. M., and Swenson, G. W. 1986, Interferometry and Synthesis in Radio Astronomy, J. Wiley and Sons, New York. 
Timbie, P. T., and Wilkinson, D. T. 1990, Ap. J., 353, 140.

Toffolatti, L., et al. 1994, Astrophys. Letters and Communications, in press.

Tucker, G. S., Griffin, G. S., Nguyen, H. T., and Peterson, J. B. 1993, Ap. J. 419, L45.

Vishniac, E. T. 1987, Ap. J., 322, 597.

Windhorst, R. A., Fomalont, E. B., Partridge, R. B., and Lowenthal, J. D. 1993, Ap. J. 405, 498.

Windhorst, R. A., Franklin, B. E., Pascarelle, S. M., Fomalont, E. B., Kellermann, K. I., Griffiths, R. E., Partridge, R. B., and Richards, E. 1995, Nature, submitted.

Wollack, E. J., et al. 1993, Ap. J. (Letters) 419, L49. 